\title{
MicroRNA-181 inhibits glioblastoma cell growth by directly targeting CCL8
}

\author{
FENGYU ZHAI $^{1,2}$, XINFENG CHEN $^{1,3}$, QIANYI HE ${ }^{4}$, HENG ZHANG $^{5}$, \\ YONGQIANG HU ${ }^{2}$, DAN WANG ${ }^{1}$, SHASHALIU ${ }^{1}$ and YI ZHANG $^{1,3,6,7}$ \\ ${ }^{1}$ Biotherapy Center, The First Affiliated Hospital of Zhengzhou University, Zhengzhou, Henan 450052; \\ ${ }^{2}$ Department of Radiotherapy, Puyang Oil Field General Hospital, Puyang, Henan 457000; Departments of ${ }^{3}$ Oncology, \\ ${ }^{4}$ Neurology and ${ }^{5}$ Radiotherapy, The First Affiliated Hospital of Zhengzhou University, Zhengzhou, Henan 450052; \\ ${ }^{6}$ School of Life Sciences, Zhengzhou University, Zhengzhou, Henan 450001; ${ }^{7}$ Engineering Key \\ Laboratory for Cell Therapy of Henan Province, Zhengzhou, Henan 450052, P.R. China
}

Received August 9, 2018; Accepted May 30, 2019

DOI: $10.3892 / \mathrm{ol} .2019 .10480$

\begin{abstract}
MicroRNAs (miRNAs/miRs), including miR-181, are closely linked to the development and progression of glioblastoma. However, the function of miR-181 in glioblastoma has not been fully clarified. The aim of the present study was to investigate the role of miR-181 in glioblastoma. miR-181 was revealed to be downregulated in glioblastoma tissues and cell lines, and associated with poor prognosis in patients with glioblastoma. Overexpression of miR-181 inhibited glioblastoma cell proliferation, invasion and migration, arrested glioblastoma cell cycle in the G1 phase and induced glioblastoma cell apoptosis. miR-181 was demonstrated to decrease expression of C-C motif chemokine ligand 8 (CCL8) by directly interacting with its 3'-untranslated region. Overexpression of CCL8 inversely reversed the proliferation, invasion and migration-promoting effects of miR-181 in glioblastoma cells. Furthermore, CCL8 was upregulated in glioblastoma tissues and was negatively correlated with miR-181 expression. These results indicate that miR-181 is a potential molecular biomarker or therapeutic target in the clinical management of glioblastoma.
\end{abstract}

\section{Introduction}

Glioblastoma is the most common primary brain malignancy in adults, with an average annual incidence of $\sim 3-10$ in every 100,000 adults in the United States (1,2). Glioblastoma is defined as any tumor that is produced by glial cells or precursor cells, including astrocytoma, glioblastoma, ependymoma, mixed glioblastoma, and other rare histologies (3). Grade IV glioblastomas have a poor

Correspondence to: Dr Yi Zhang, Biotherapy Center, The First Affiliated Hospital of Zhengzhou University, 1 Jianshe East Road, Zhengzhou, Henan 450052, P.R. China

E-mail: yizhang@zzu.edu.cn

Key words: microRNA-181, C-C motif chemokine ligand 8, proliferation, invasion, migration, cell cycle, apoptosis prognosis, with a median survival time of 14.6-16 months and a 5 -year survival rate of $3.4 \%(4,5)$. The standard first-line treatment for glioblastoma involves a variety of modalities, including surgical resection, radiotherapy and temozolomide-adjuvant chemotherapy (6). Despite surgical resection and chemoradiation, tumors in almost all patients are likely to relapse, with a 2-year survival rate of only $26 \%$ following relapse (7). There is currently no standard and uniform management method for recurrent glioblastoma. Therefore, the search for novel glioblastoma treatment pathways is particularly important. In recent years, with the development of molecular biology techniques, tumor-related genetic research has increased (8). At present, gene therapies and molecular-targeted drugs are a novel area of study $(9,10)$. The most important aspect of these treatments is identifying a specific molecular therapy target for different tumors.

microRNAs (miRNAs/miRs) have been identified as promising therapeutic targets in a number of types of cancer $(11,12)$. miRNAs are short endogenous single-stranded RNA molecules that transcriptionally inhibit gene expression by targeting 3'-untranslated regions (3'-UTRs) of messenger RNAs (mRNAs) by binding to complementary sequences $(13,14)$. miRNAs regulate a number of important cellular functions, including angiogenesis, proliferation, apoptosis and invasion, and are deregulated in numerous types of cancer (15). A growing body of research indicates that miRNAs regulate the expression of chemokines, further effecting disease progression and prognosis (16). miRNA-181 has been found to inhibit the progression of cervical cancer by targeting a transcription factor, yin-yang-1 $(17,18)$. The expression of miR-181 and its impact on CCL8 in the progression of glioblastoma has not been studied.

The present study aimed to evaluate the potential of miRNA-181 and CLL8 as biomarkers or treatment targets in glioblastoma by investigating their expression in patients, and to determine the effects of altered miRNA-181 and CCL8 expression in glioblastoma cell lines.

\section{Materials and methods}

Patient tissues. The present study was approved by the Institutional Review Board and Ethics Committee of 
The First Affiliated Hospital of Zhengzhou University (Zhengzhou, Henan, China). Tissue samples of glioblastoma, including 23 grade II tumors, 26 grade III tumors and 31 grade IV tumors, and adjacent non-cancerous tissues samples were obtained from 80 patients with glioblastoma undergoing surgery at The First Affiliated Hospital of Zhengzhou University between February and August 2013 (46 men and 34 women; age range, 28-73 years). None of the patients had received radiotherapy, chemotherapy or other anticancer treatment prior to surgery. All participants had provided written informed consent. The patients were followed up for 3 years. The tumor tissues, including 42 tumors with diameter $<5 \mathrm{~cm}$ and 38 tumors with diameter $\geq 5 \mathrm{~cm}$, and adjacent non-tumor tissues were collected at surgery, immediately frozen in liquid nitrogen and stored at $-80^{\circ} \mathrm{C}$ until RNA or protein extraction.

Cell culture. Human glioblastoma cell lines, U251, A172 and U87 cells (cat. no. HTB-14, glioblastoma of unknown origin) and normal human astrocytes (NHA) were obtained from the American Type Culture Collection (ATCC; Manassas, VA, USA) and cultured in Dulbecco's modified Eagle's medium (DMEM; Thermo Fisher Scientific, Inc., Waltham, MA, USA) supplemented with $10 \%$ fetal bovine serum (FBS; Gibco; Thermo Fisher Scientific, Inc.). All cell lines were routinely passaged as monolayer cultures at $37^{\circ} \mathrm{C}$ in a humidified atmosphere of $5 \% \mathrm{CO}_{2}$.

Vector construction and transfection. The wild-type 3'UTR of CCL 8 mRNA and a mutated version flanked by KpnI and HindIII restriction sites were synthesized and ligated into the pGL3 luciferase reporter vector (Sangon Biotech Co., Ltd., Shanghai, China). The cDNA sequence of CCL-8 was ligated into a pcDNA3.1 (+) vector (Thermo Fisher Scientific, Inc.). The CCL-8-containing vector $(10 \mu \mathrm{M})$ and an miR-181 mimic (100 nM) (Shanghai GenePharma Co., Ltd., Shanghai, China) were transfected into U87 and A172 cells using Lipofectamine ${ }^{\circledR}$ 2000 (Thermo Fisher Scientific, Inc.) according to the manufacturer's protocol. The sequences were as follows: miR-181 mimic, 5'-AACAUUCAACGCUGUCGGUGAGUUCAC CGACAGCG-3' and miR-negative control (NC), 5'-UUCUCC GAACGUGUCACGUTT-3'. Subsequent experiments were performed $48 \mathrm{~h}$ following transfection.

Dual-luciferase reporter assay. Bioinformatics analysis was conducted to predict the target gene using TargetScan (version 6.2; http://genes.mit.edu/targetscan). A luciferase reporter gene assay was performed in cells $48 \mathrm{~h}$ following transfection with the pGL3 vector containing the wild-type or mutated 3'-UTR of CCL8. The Dual-Luciferase ${ }^{\circledR}$ Reporter Assay System (Promega Corporation, Madison, WI, USA) was used according to the manufacturer's instructions. Renilla luciferase was co-transfected as a control for normalization.

Reverse transcription-quantitative polymerase chain reaction $(R T-q P C R)$. Total RNA from all samples and cell lines was extracted using TRIzol ${ }^{\circledR}$ (Invitrogen; Thermo Fisher Scientific, Inc.). Isolated RNA was reverse-transcribed using MultiScribe reverse transcriptase (Applied Biosystems; Thermo Fisher Scientific, Inc). Briefly, samples containing $1 \mu \mathrm{g}$ total RNA were incubated with $1 \mu \mathrm{l}$ gDNA Eraser, $2 \mu 1$ 5X gDNA eraser buffer and RNase free $\mathrm{dH}_{2} \mathrm{O}$ at $42^{\circ} \mathrm{C}$ for $2 \mathrm{~min}$. After adding the enzyme mix, the reaction was incubated at $37^{\circ} \mathrm{C}$ for $15 \mathrm{~min}$. cDNA was diluted and stored at $-20^{\circ} \mathrm{C}$ prior to use. mRNA expression was detected with SYBR ${ }^{\circledR}$ Premix Ex Taq II (Takara Biotechnology Co., Ltd., Dalian, China). The PCR primers were designed as follows: miR-181, forward 5'-AACATTCAACGCTGTCGG TGAAGT-3', reverse 5'-ACTTCACCGACAGCGTTGAAT GTT-3'; U6, forward 5'-CTCGCTTCGGCAGCACA-3', reverse 5'-AACGCTTCACGAATTTGCGT-3'; CCL-8 forward 5'-AGA TGAAGGTTTCTGCAGCGC-3', reverse 5'-TGGAAACTG AATCTGGCTGAG-3'; and GAPDH, forward 5'-GCACCG TCAAGGCTGAGAAC-3' and reverse 5'-TGGTGAAGACGC CAGTGGA-3'. RT-qPCR reactions were performed as follows: $95^{\circ} \mathrm{C}$ for $10 \mathrm{~min}$ followed by 40 cycles of $95^{\circ} \mathrm{C}$ for $25 \mathrm{sec}$ and $60^{\circ} \mathrm{C}$ for $30 \mathrm{sec}$. The relative expression levels of miR-181 and CCL-8 relative to GAPDH and U6 were calculated using the relative quantification $2^{-\Delta \Delta \mathrm{Cq}}$ method (19). Each sample was assayed in triplicates. The high and low expression of miR-181 was defined according to the average expression levels of miR-181 in patients with glioblastoma.

Western blot analysis. Total protein was extracted from the tissues and cells using radioimmunoprecipitation lysis buffer (Beyotime Institute of Biotechnology, Jiangsu, China) and separated using SDS-PAGE (10\% gel), and then transferred to polyvinylidene difluoride membranes. The membranes were then incubated with $5 \%$ non-fat milk in Tris-buffered saline (TBS; 20 mM Tris- $\mathrm{HCl}, \mathrm{pH} 7.5,150 \mathrm{mM} \mathrm{NaCl}$ ) containing $0.1 \%$ Tween 20 at room temperature. Subsequently, the membranes were incubated at room temperature with rabbit antibodies against CCL-8 (cat. no. ab39625; 1:500; Abcam, Cambridge, UK;) and GAPDH (cat. no. sc-47724; 1:500; Santa Cruz, Biotechnology, Inc., Dallas, TX, USA) overnight at $4^{\circ} \mathrm{C}$. Membranes were washed with TBST and incubated at room temperature with horseradish peroxidase-conjugated goat anti-rabbit secondary antibody (cat. no. 7074; 1:5,000; Cell Signaling Technology, Inc., Danvers, MA, USA) for $40 \mathrm{~min}$. Protein bands were detected by enhanced chemiluminescence (ECL) using Pierce ECL Western Blot Substrate (Thermo Fisher Scientific, Inc.) and exposed to an X-ray film using an ECL detection system (Thermo Fisher Scientific, Inc.). GAPDH served as a loading control. All assays were performed in triplicate.

Cell proliferation assay. A total of $\sim 1 \times 10^{3}$ cells/well were cultured in 96-well plates and a Cell Counting Kit-8 (CCK-8; Dojindo Molecular Technologies, Inc., Kumamoto, Japan) was used to determine proliferation in each treatment group (6 wells per group) and in the blank controls. Cells were incubated with $10 \mu \mathrm{l} \mathrm{CCK}-8$ at different time points $(0,24,48$ and $72 \mathrm{~h})$ for $3 \mathrm{~h}$ at $37^{\circ} \mathrm{C}$ and $5 \% \mathrm{CO}_{2}$. The absorbance value at $450 \mathrm{~nm}$ was measured. The cell growth curves were plotted using the absorbance value at each time point. All assays were performed in triplicate.

Migration and invasion assay. Transwell chambers (24-wells; Corning Inc., Corning, NY, USA) were used to evaluate the migratory and invasive ability of glioblastoma cells. Transfected U87 and A172 cells in DMEM were added to the top chambers 

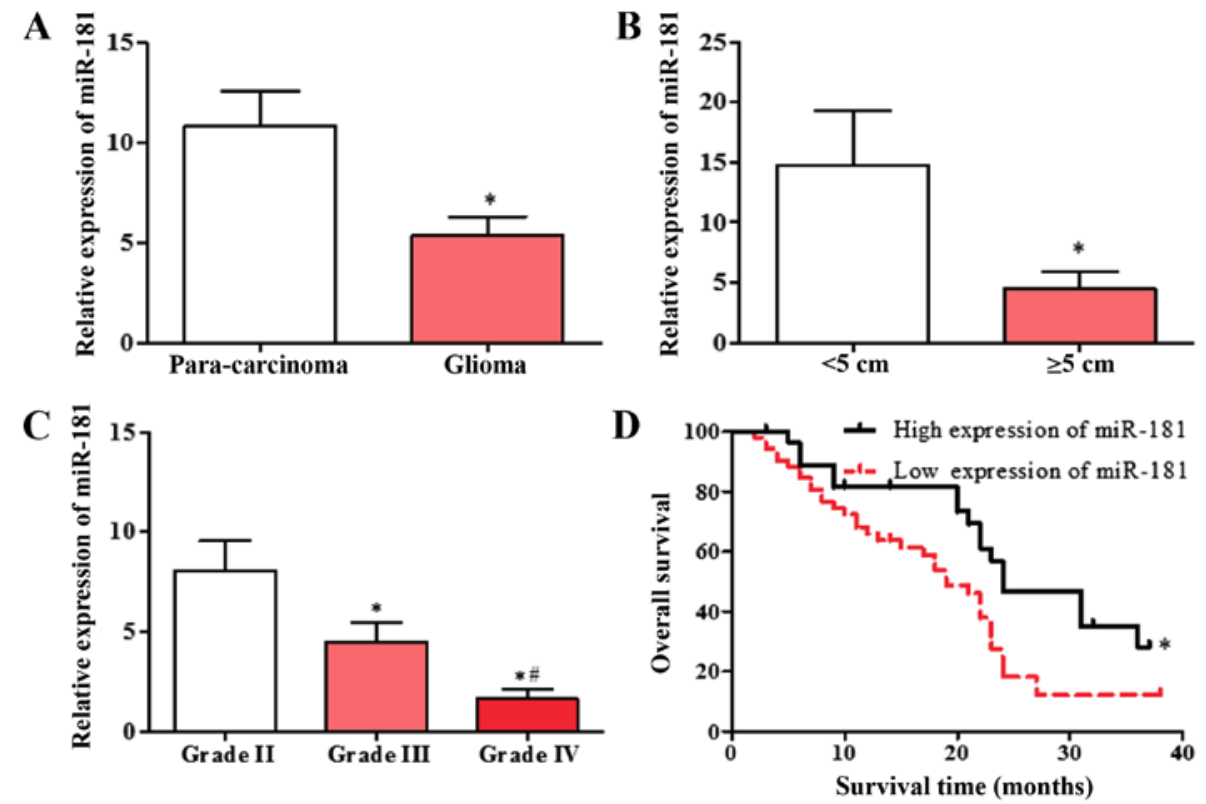

Figure 1. Downregulation of miR-181 in glioblastoma and para-carcinoma tissues. (A) Expression of miR-181 in glioblastoma and adjacent tissue. (B) Expression of miR-181 in glioblastoma tumors with diameter of $<5$ or $\geq 5 \mathrm{~cm}$. (C) Expression of miR-181 in glioblastoma tumors of different grades. (D) Expression of miR-181 was associated with poor prognosis in patients with glioblastoma (low expression of miR-181, n=52; high expression of miR-181, $n=28$ ). Data are presented as the mean \pm standard deviation and based on at least three independent experiments. ${ }^{*} \mathrm{P}<0.05 .{ }^{~} \mathrm{P}<0.05$ vs. grade IV. miR-181, microRNA-181.

of the Transwell plate that were pre-coated with Matrigel (BD Biosciences, San Jose, CA, USA) for the invasion assay, and all other steps were identical. A total of $4 \times 10^{4}$ transfected cells without FBS were placed in the top chamber, and the bottom chamber was filled with $20 \%$ FBS. The cells were incubated at $37^{\circ} \mathrm{C}$ for $24 \mathrm{~h}$ to allow migration or invasion through the membrane. The cells were then stained at room temperature with crystal violet (Beyotime Institute of Biotechnology, Shanghai, China) for $10 \mathrm{~min}$. All assays were performed in triplicates.

Flow cytometry. Cells were cultured in 6-well plates at a concentration of $3 \times 10^{5}$ cells/well. Cells were washed with PBS and harvested with $0.25 \%$ trypsin without EDTA. Cells were then washed with PBS and stained with $5 \mu$ 1 Annexin V-fluorescein isothiocyanate (BD Biosciences) and $5 \mu \mathrm{l}$ propidium iodide (BD Biosciences) for $15 \mathrm{~min}$ at room temperature in the dark according to the manufacturer's instructions. Data acquisition and analyses were performed on a BD FACSCalibur flow cytometer (Becton, Dickinson and Company, Franklin Lakes, NJ, USA) using CellQuest software (version 5.2.1; BD Biosciences). All assays were performed in triplicate.

Statistical analysis. Data are shown as the mean \pm standard deviation where applicable. Graphpad Prism (version 5.0; Graphpad Software Inc., La Jolla, CA, USA) was used for all statistical analysis. The differences between groups were evaluated by one way analysis of variance followed by Dunnett's test. Kaplan-Meier analysis and a log-rank test were used to compare patient survival. The correlation between the expression levels of miR-181 and CCL8 was assessed using Spearman's correlation analysis. $\mathrm{P}<0.05$ was considered to indicate a statistically significant difference.

\section{Results}

miR-181 is downregulated in patients with glioblastoma and is associated with poor prognosis. Expression of miR-181 in glioblastoma and adjacent tissues was detected using RT-qPCR. miR-181 expression in glioblastoma tissues was significantly decreased compared with that in adjacent tissues (Fig. 1A), and the expression of miR-181 in glioblastoma with tumor diameter $\geq 5 \mathrm{~cm}$ was decreased compared with that in glioblastoma with tumor diameter $<5 \mathrm{~cm}$ (Fig. 1B). The expression of miR-181 in patients with different diagnostic grades was also evaluated. The expression of miR-181 in patients with grade III and IV glioblastoma was significantly lower compared with that in patients with grade II, and the expression of miR-181 in patients with grade IV glioblastoma was significantly lower compared with that in patients with grade III (Fig. 1C). The patient group with low expression of miR-181 had a significantly lower survival rate compared with the group with high expression of miR-181 (Fig. 1D). The results indicate that decreased expression of miR-181 is associated with poor prognosis.

Overexpression of miR-181 inhibited cell proliferation, invasion and migration in glioblastoma cell lines. The expression of miR-181 in human glioblastoma cell lines U251, A172 and U87, and normal human astrocytes NHA was detected by RT-qPCR. The expression of miR-181 in glioblastoma cell lines was significantly lower compared with that in normal human astrocytes NHA cells (Fig. 2A). Following transfection of A172 and U87 cells with miR-181 mimic, miR-181 expression was significantly increased compared with that in cells transfected with miR-NC (Fig. 2B). The effect of miR-181 on proliferation in glioblastoma cells, was investigated using the CCK-8 assay. The proliferation of A172 and U87 cells 
A
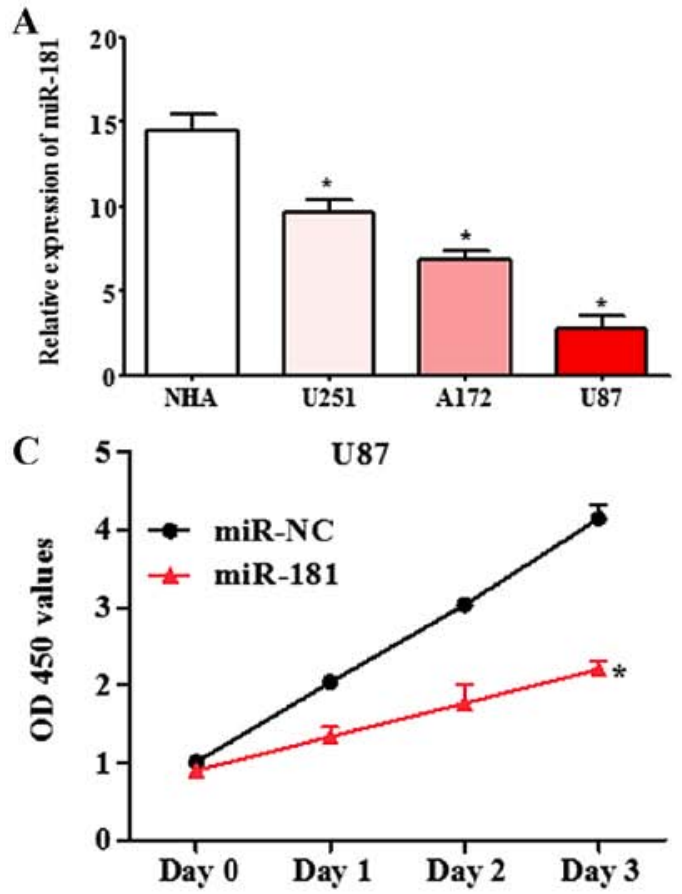

E

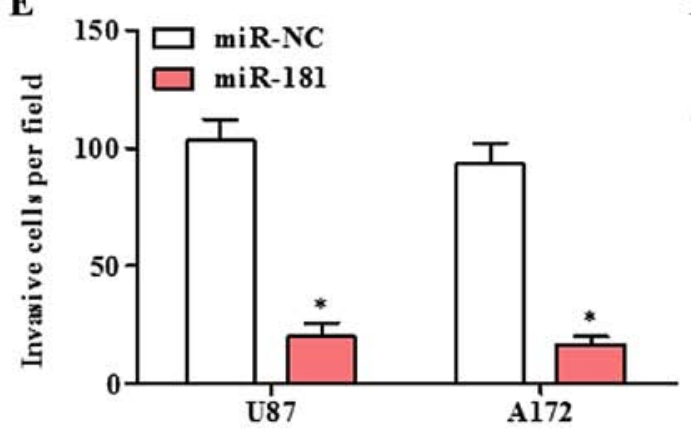

G

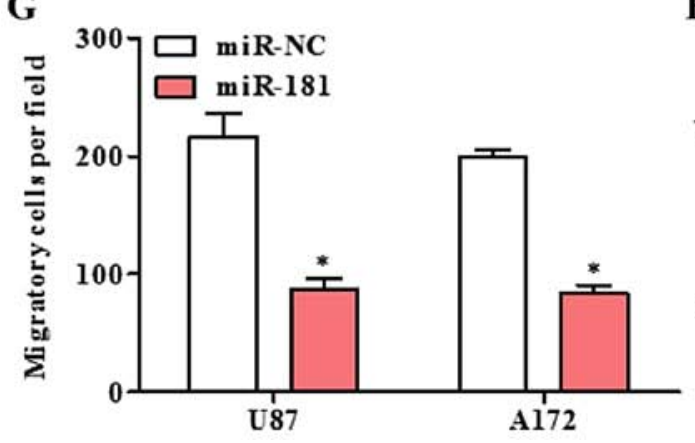

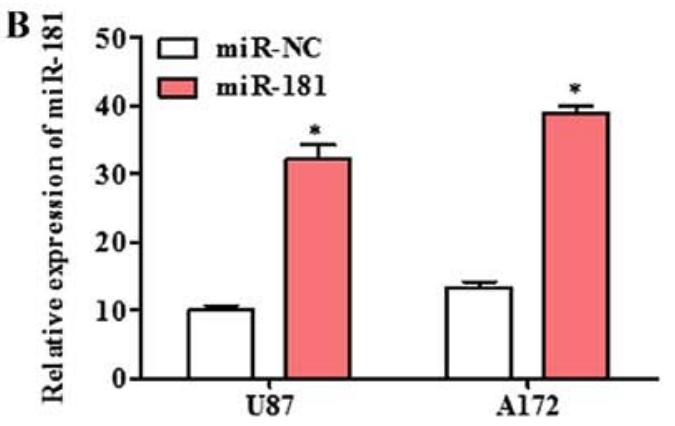

D

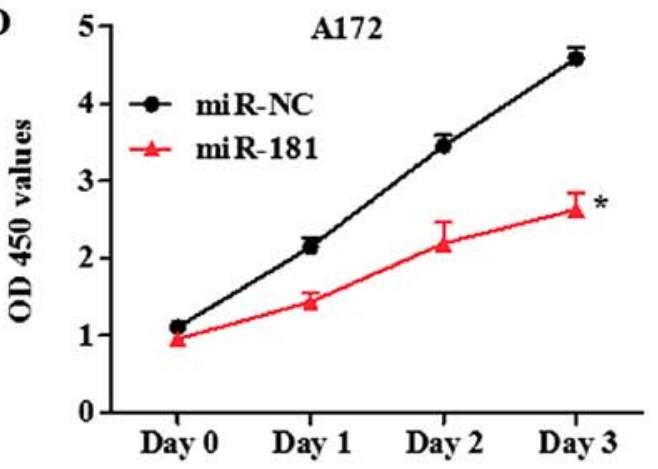

F

U87

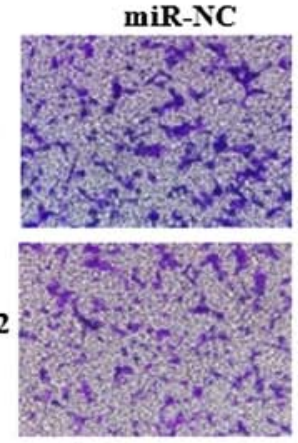

miR-NC
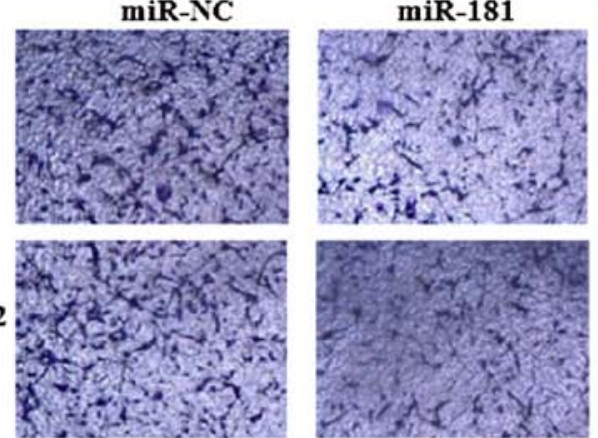

Figure 2. Proliferation, migration and invasion in glioblastoma cell lines with overexpression of miR-181. (A) Expression of miR-181 in U251, A172 and U87 glioblastoma lines was reduced compared with that in NHA cells. (B) Expression of miR-181 in U87 and A172 cells transfected with miR-181 and miR-NC. (C) Proliferation of U87 cells transfected with miR-181 and miR-NC. (D) Proliferation of A172 cells transfected with miR-181 and miR-NC. (E and F) Invasion of U87 and A172 cells transfected with miR-181 and miR-NC. (G and H) Migration of U87 and A172 cells transfected with miR-181 and miR-NC, (magnification $\mathrm{x} 100)$. Data are presented as the mean \pm standard deviation and based on at least three independent experiments. " $\mathrm{P}<0.05$. miR-181, microRNA-181; miR-NC, miR-negative control; NHA, normal human astrocytes; OD, optical density.

transfected with miR-181 was decreased compared with that of cells transfected with miR-NC (Fig. 2C and D). A Transwell assay revealed that the ability of A172 and U87 cells transfected with miR-181 to invade or migrate across a membrane was decreased compared with that of cells transfected with miR-NC (Fig. 2E-H). The results indicate that upregulation of miR-181 inhibits glioblastoma cell growth, invasion and migration.
Overexpression of miR-181 arrested cell cycle in the G1 phase and induced cell apoptosis in glioblastoma cell lines. The effects of overexpression of miR-181 on cell cycle and apoptosis in glioblastoma cell was evaluated. The cell cycle was significantly shifted from S phase to G1 phase in U87 and A172 cells transfected with miR-181 compared with those transfected with miR-NC (Fig. 3A-D). The percentage of cells in the G1 phase was increased and the percentage 

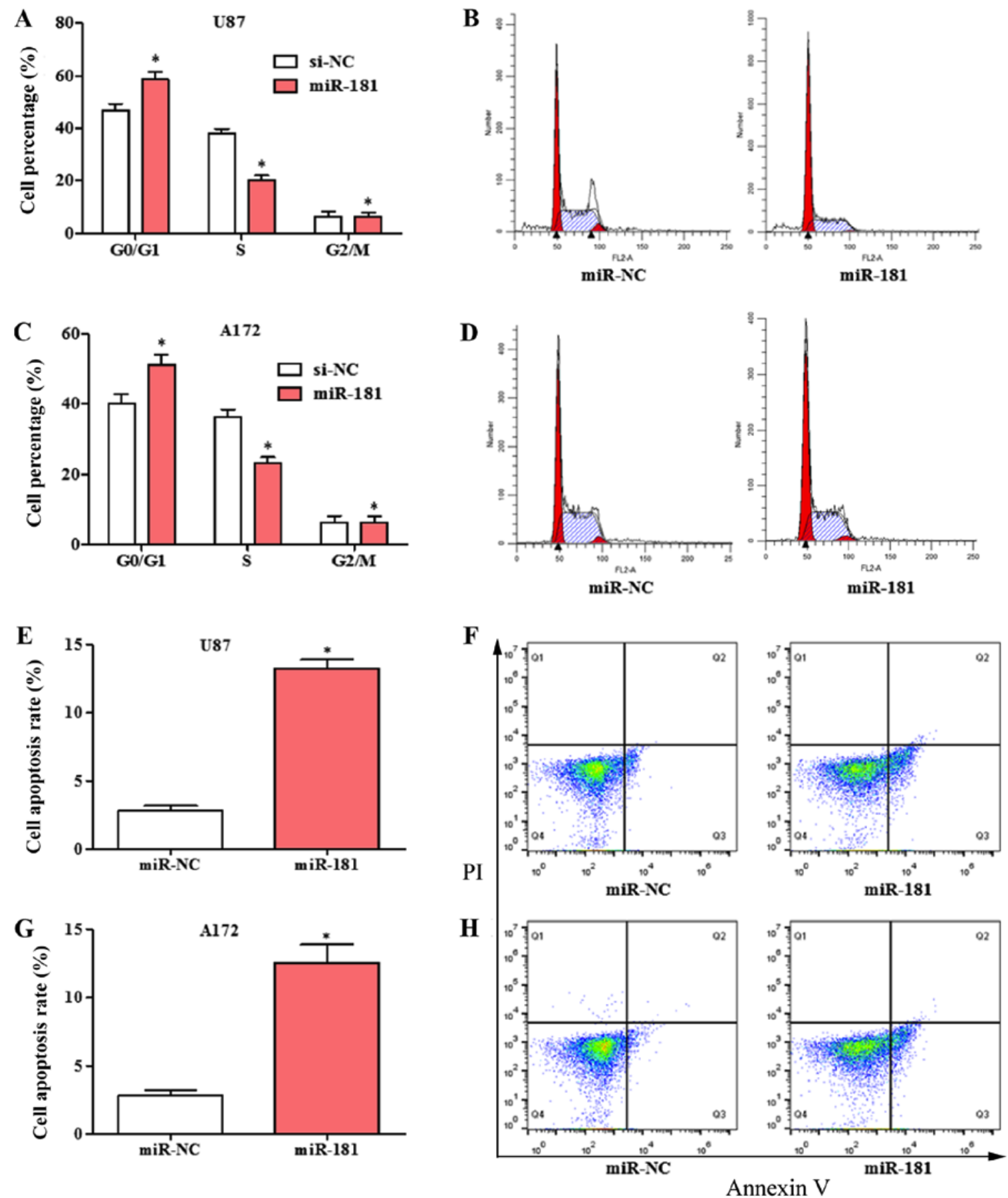

Figure 3. Cell cycle arrest and apoptosis in glioblastoma cell lines with overexpression of miR-181. (A and B) Cell cycle distribution in U87 cells transfected with miR-181 and miR-NC. (C and D) Cell cycle distribution in A172 cells transfected with miR-181 and miR-NC. (E and F) Apoptosis rate in U87 cells transfected with miR-181 and miR-NC. (G and H) Apoptosis rate in A172 cells transfected with miR-181 and miR-NC. All data are shown as the mean \pm standard deviation and based on at least three independent experiments. "P<0.05. miR-181, microRNA-181; miR-NC, miR-negative control; PI, propidium iodide.

in the $\mathrm{S}$ phase was decreased in the miR-181-overexpressing groups. Overexpression of miR-181 promoted apoptosis in U87 (Fig. 3E and F) and A172 cells (Fig. 3G and H).

CCL8 is a target of miR-181. The sequence alignment of miR-181 and the 3'-UTR of CCL-8 mRNA is presented in Fig. 4A. To further determine the interaction between miR-181 and CCL-8, luciferase vectors containing wide-type or mutated CCL8 3'-UTR were co-transfected into 293 cells (ATCC) with miR-NC or miR-181. The dual-luciferase reporter assay revealed that transfection with miR-181 reduced the activity of the luciferase reporter fused to the wild-type 3'-UTR of CCL-8, but did not suppress that of the reporter fused to the mutated version (Fig. 4B). Following transfection with miR-181, protein and mRNA expression of CCL8 in U87 and A172 cells was decreased compared to those of cells transfected with miR-NC (Fig. 4C and D). Following transfection of A172 and U87 cells with pcDNA-CCL8, CCL8 mRNA expression was significantly higher compared with cells that were transfected with pcDNA (Fig. 4E). The results indicated 

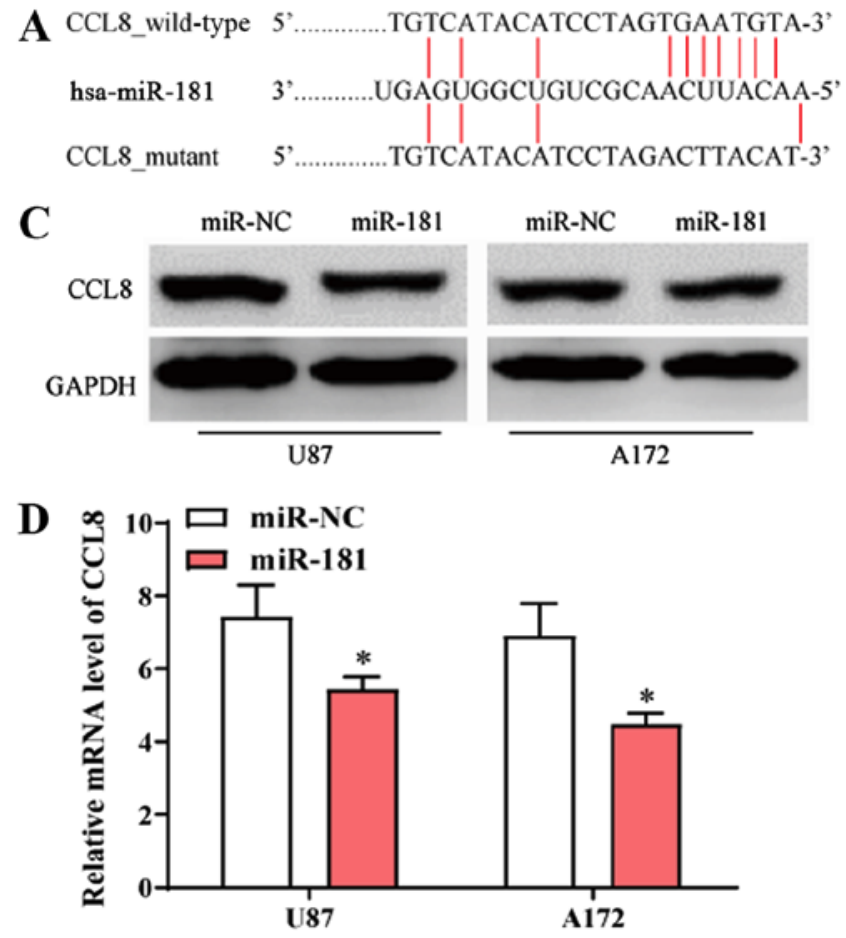

B

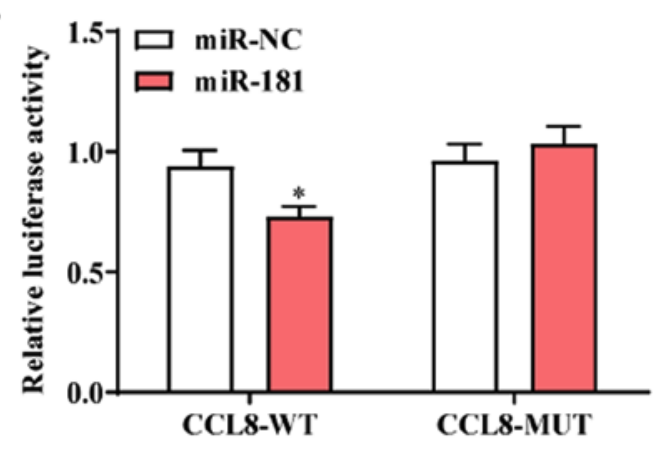

E

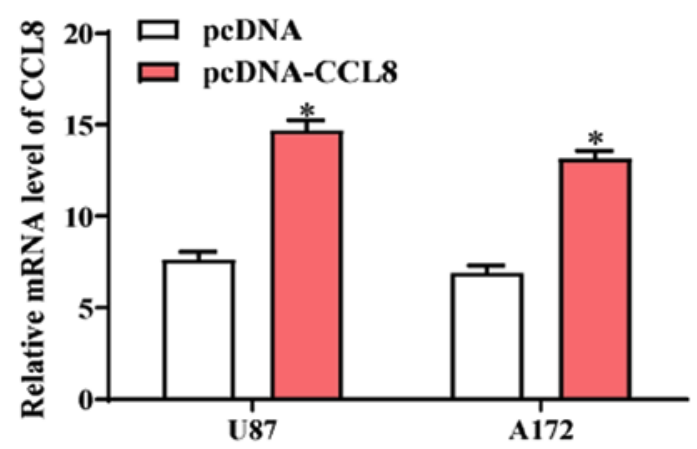

Figure 4. Identification of CCL8 as a direct target of miR-181. (A) Predicted binding sites of miR-181 in the wild-type and mutant 3'-UTR of CCL8. (B) Luciferase activity assay of cells containing wide-type or mutant CCL8-3'-UTR following transfection with miR-181 or miR-NC. (C) Protein expression of CCL8 in U87 and A172 cells transfected with miR-181 or miR-NC. (D) mRNA expression of CCL8 in U87 and A172 cells transfected with miR-181 or miR-NC. (E) Protein expression of CCL8 in U87 and A172 cells transfected with pcDNA-CCL8 and pcDNA. All data are shown as the mean \pm standard deviation and based on at least three independent experiments. ${ }^{*} \mathrm{P}<0.05$. miR-181, microRNA-181; UTR, untranslated region; CCL8, C-C motif chemokine ligand 8; miR-NC, miR-negative control.

that CCL8 was a direct target of miR-181 and overexpression of miR-181 reduced the expression of CCL8.

Overexpression of CCL8 partially reversed the effects of miR-181 overexpression in glioblastoma cell lines. To confirm that the effects of miR-181 on cell proliferation, invasion and migration are mediated by CCL8 downregulation, U87 and A172 cells were transfected with miR-NC, miR-181 and co-transfected with CCL8. Overexpression of CCL8 partially reversed the inhibition of proliferation by miR-181 in U87 and A172 cells (Fig. 5A and B). The inhibition of invasion and migration by miR-181 in the two cell lines was also partially rescued by overexpression of CCL8 (Fig. 5C and D). Flow cytometric analysis revealed that overexpression of CCL8 partially reversed the apoptotic effect of miR-181 in U87 and A172 cells (Fig. 5E and F). These results suggested that miR-181 promoted glioblastoma cell proliferation, invasion and migration and inhibited apoptosis partially through downregulation of CCL8.

Expression of CCL8 in glioblastoma tissues was negatively correlated with miR-181 expression. The expression of CCL8 in glioblastoma and adjacent tissues were detected by RT-qPCR. CCL8 expression in glioblastoma tissues was significantly higher compared with that in adjacent tissues (Fig. 5G). Spearman's correlation analysis showed that CCL8 was negatively correlated with miR-181 expression (Fig. 5H). The results further suggested that miR-181 may inhibit glioblastoma cell growth and induces apoptosis by directly targeting CCL8.

\section{Discussion}

In the present study, miR-181 expression was revealed to be low in glioblastoma tissue and associated with poor prognosis in patients with glioblastoma. Overexpression of miR-181 inhibited glioblastoma cell proliferation, invasion and migration and arrested glioblastoma cell cycle in the G1 phase and induced glioblastoma cell apoptosis. CCL8 was upregulated in glioblastoma tissues and was negatively correlated with miR-181 expression. CCL8 was confirmed as a direct target of miR-181 using a dual luciferase assay. In addition, overexpression of CCL8 reversed the inhibition of proliferation, invasion and migration and induction of apoptosis by miR-181 in glioblastoma cells. The results suggested that downregulated miR-181 may promote glioblastoma cell proliferation, invasion and migration and inhibit apoptosis partially through binding and inhibiting CCL8.

miRNAs can regulate cell proliferation, invasion, migration, the cell cycle and apoptosis (20), as well as regulating transcription through RNA polymerase, to impact tumor development $(21,22)$. Previous studies indicated that aberrant expression of miRNAs has been identified in a number of types of cancer, including stomach, liver, colorectal, breast and cervical cancer, as well as glioma and others $(23,24)$. miR-181 is an important miRNA that is involved in the development of multiple tumor types $(25,26)$. miR-181 was found to regulate cisplatin-resistant non-small cell lung cancer via the PTEN/PI3K/AKT pathway (27). In the present study, downregulation of miR-181 was identified in patients with 

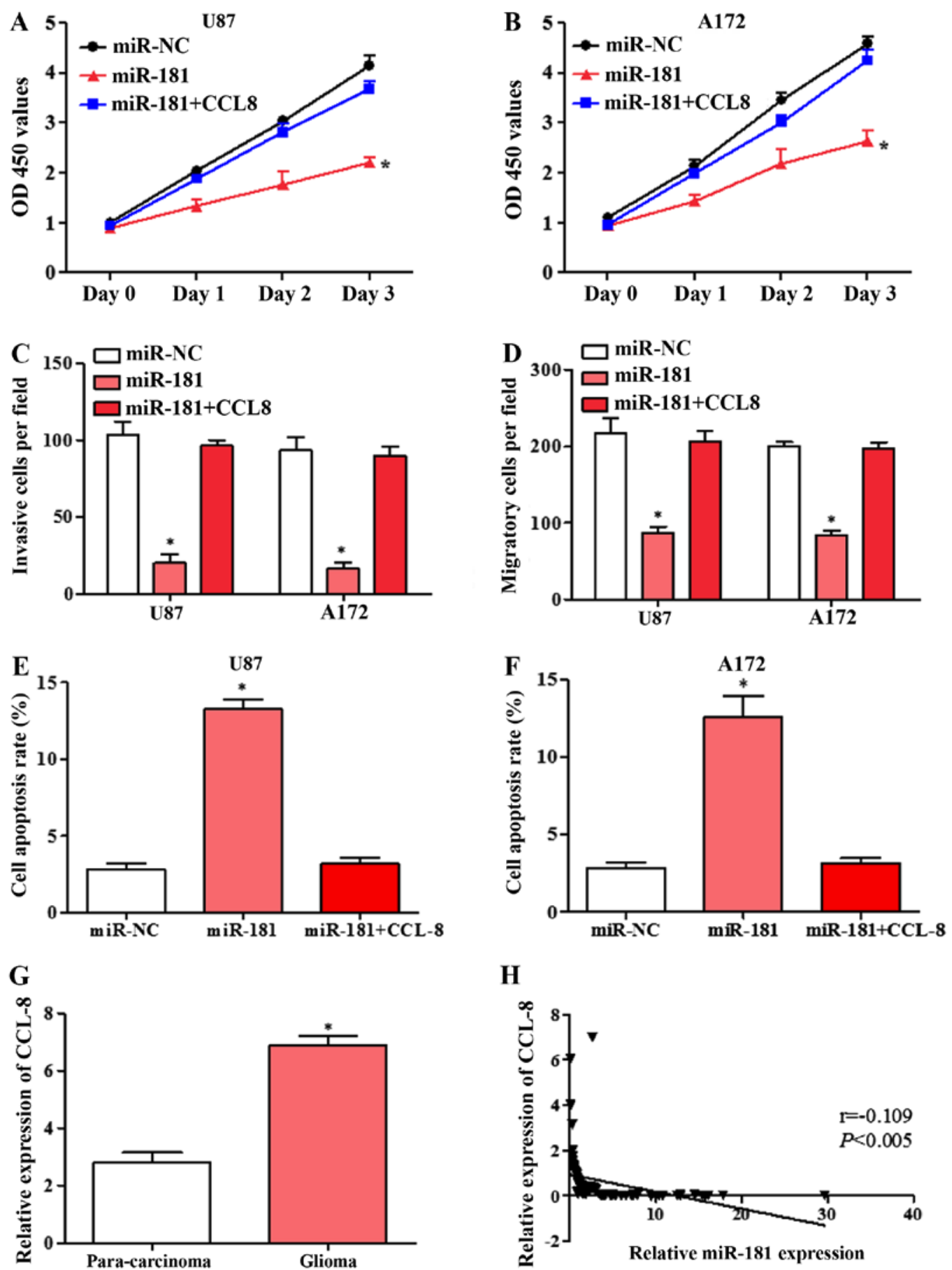

Figure 5. Overexpression of CCL8 reverses the miR-181-mediated inhibition of proliferation, invasion and migration and induction of apoptosis in glioblastoma cell lines. (A) Proliferation of U87 cells transfected with miR-NC, miR-181 and co-transfected with miR-181 and CCL8. (B) Proliferation of A172 cells transfected with miR-NC, miR-181 and co-transfected with miR-181 and CCL8. (C) Invasion of U87 and A172 cells transfected with miR-NC, miR-181 and co-transfected with miR-181 and CCL8. (D) Migration of U87 and A172 cells transfected with miR-NC, miR-181 and co-transfected with miR-181 and CCL8. (E) Apoptosis rate of U87 cells transfected with miR-NC, miR-181 and co-transfected with miR-181 and CCL8; (F) apoptosis rate of A172 cells transfected with miR-NC, miR-181 and co-transfected with miR-181 and CCL8. (G) Expression of CCL8 in glioblastoma and para-carcinoma tissue detected by RT-qPCR $(\mathrm{n}=80)$. $(\mathrm{H})$ Expression of CCL8 in glioblastoma negatively correlated with miR-181 expression, analyzed by Spearman's correlation analysis $(\mathrm{n}=80)$. All data are shown as the mean \pm standard deviation and based on at least three independent experiments. ${ }^{*} \mathrm{P}<0.05$. miR-181, microRNA-181; CCL8, C-C motif chemokine ligand 8; OD, optical density; RT-qPCR, reverse transcription-quantitative polymerase chain reaction; miR-NC, miR-negative control.

glioblastoma, and was associated with poor prognosis. The detailed mechanism remains unknown and further research is required.

To explore the function of miR-181 in glioblastoma, the proliferation, invasion and migratory abilities of A172 and U87 cells transfected with miR-181 were investigated and found to be lower compared with cells transfected with miR-NC. In addition, the cell cycle was significantly shifted from $\mathrm{S}$ phase to $\mathrm{G} 1$ phase, and apoptosis was promoted in U87 and A172 cells transfected with miR-181, compared with cells transfected with miR-NC. Su et al (28) reported that miR-181 is aberrantly overexpressed in patients with acute myeloid 
leukemia and can inhibit granulocytic and macrophage-like differentiation by directly targeting and downregulating the expression of PRKCD, CTDSPL and CAMKK1.

CCL8 has been reported to be a CCR5 agonist with a similar affinity to the receptor as CCL3 (MIP-1a), which in combination with CCR5 stimulates receptor internalization and intracellular calcium release $(29,30)$. It has been reported that in lung cancer, CCL8 further promotes tumor progression by recruiting CCR5+ Treg cells to the tumor site (27). In patients with malignant melanoma, CCL8 also promotes tumorigenesis and progression (31). At present, the expression of CCL8 in glioma tissue has not been explored, and its role in the progression of glioma and the corresponding molecular mechanisms remain unclear. In the present study, the dual-luciferase reporter assay indicated that miR-181 reduced the activity of the luciferase reporter fused to the 3'-UTR-WT of CCL-8 but did not suppress that of the reporter fused to the MUT version. Protein and mRNA expression of CCL8 were significantly decreased in U87 and A172 cells transfected with miR-181. These results suggest that CCL8 is a direct target of miR-181 and overexpression of miR-181 reduces the expression of CCL8.

The impact of miR-181 via CCL8 on cell proliferation, invasion and migration was further confirmed by overexpression of CCL8. U87 and A172 cells were co-transfected with miR-181 and CCL8. The results demonstrated that overexpression of CCL8 partially reversed the proliferation, invasion, migration and apoptosis effects of miR-181 in U87 and A172 cells. In addition, CCL8 expression was high in glioblastoma tissues and negatively correlated with miR-181 expression. The results further suggested that miR-181 inhibits glioblastoma cell growth and induces apoptosis by directly targeting CCL8.

In summary, the present study revealed that miR-181 was downregulated in glioblastoma and associated with poor prognosis. Overexpression of miR-181 inhibited proliferation, invasion and migration and induced apoptosis in glioblastoma cells by directly targeting CCL8. miR-181 may serve as a novel prognostic biomarker glioblastoma.

\section{Acknowledgements}

Not applicable.

\section{Funding}

No funding was received.

\section{Availability of data and materials}

All data generated or analyzed during the present study are included in this published article.

\section{Authors' contributions}

$\mathrm{YZ}$ and FZ conceived and designed the study. FZ and $\mathrm{XC}$ performed the majority of experiments. QH, HZ and $\mathrm{YH}$ collected the clinical samples. DW and SL helped to perform the cell culture experiments. YZ and FZ wrote the manuscript. All authors have read and approved this manuscript.

\section{Ethics approval and consent to participate}

The present study was approved by the Ethics Committees of The First Affiliated Hospital of Zhengzhou University. Written informed consent was provided by all patients.

\section{Patients consent for publication}

Not applicable.

\section{Competing interests}

The authors declare that they have no competing interests.

\section{References}

1. Vengoji R, Macha MA, Batra SK and Shonka NA: Natural products: A hope for glioblastoma patients. Oncotarget 9: 22194-22219, 2018.

2. Ouanouki A, Lamy S and Annabi B: Periostin, a signal transduction intermediate in TGF- $\beta$-induced EMT in U-87MG human glioblastoma cells, and its inhibition by anthocyanidins. Oncotarget 9: 22023-22037, 2018.

3. Liu J, Wang WM, Zhang XL, Du QH, Li HG and Zhang Y: Effect of downregulated lncRNA NBAT1 on the biological behavior of glioblastoma cells. Eur Rev Med Pharmacol Sci 22: 2715-2722, 2018.

4. Jayachandran A, Jonathan GE, Patel B and Prabhu K: Primary spinal cord glioblastoma metastasizing to the cerebellum: A missed entity. Neurol India 66: 854-857, 2018.

5. Huang L, Boling W and Zhang JH: Hyperbaric oxygen therapy as adjunctive strategy in treatment of glioblastoma multiforme. Med Gas Res 8: 24-28, 2018.

6. Paulmurugan R, Afjei R, Sekar TV, Babikir HA and Massoud TF: A protein folding molecular imaging biosensor monitors the effects of drugs that restore mutant p53 structure and its downstream function in glioblastoma cells. Oncotarget 9: 21495-21511, 2018.

7. Tanaka H, Yamaguchi T, Hachiya K, Miwa K, Shinoda J, Hayashi M, Ogawa S, Nishibori H, Goshima S and Matsuo M: ${ }^{11} \mathrm{C}$-methionine positron emission tomography for target delineation of recurrent glioblastoma in re-irradiation planning. Rep Pract Oncol Radiother 23: 215-219, 2018.

8. Puchalski RB, Shah N, Miller J, Dalley R, Nomura SR, Yoon JG, Smith KA, Lankerovich M, Bertagnolli D, Bickley K, et al: An anatomic transcriptional atlas of human glioblastoma. Science 360: 660-663, 2018.

9. Schulze M, Hutterer M, Sabo A, Hoja S, Lorenz J, Rothhammer-Hampl T, Herold-Mende C, Floßbach L, Monoranu C and Riemenschneider MJ: Chronophin regulates active vitamin B6 levels and transcriptomic features of glioblastoma cell lines cultured under non-adherent, serum-free conditions. BMC Cancer 18: 524, 2018.

10. Di Sebastiano AR, Deweyert A, Benoit S, Iredale E, Xu H, De Oliveira C, Wong E, Schmid S and Hebb MO: Preclinical outcomes of intratumoral modulation therapy for glioblastoma. Sci Rep 8: 7301, 2018.

11. Wang X, Sun S, Tong X, Ma Q, Di H, Fu T, Sun Z, Cai Y, Fan W, Wu Q, et al: MiRNA-154-5p inhibits cell proliferation and metastasis by targeting PIWIL1 in glioblastoma. Brain Res 1676: 69-76, 2017.

12. Hinske LC, Heyn J, Hübner M, Rink J, Hirschberger S and Kreth S: Intronic miRNA-641 controls its host Gene's pathway $\mathrm{PI} 3 \mathrm{~K} / \mathrm{AKT}$ and this relationship is dysfunctional in glioblastoma multiforme. Biochem Biophys Res Commun 489: 477-483, 2017.

13. Zhang X,Zhang X, Hu S, Zheng M,Zhang J,Zhao J,Zhang X, Yan B, Jia L, Zhao J, et al: Identification of miRNA-7 by genome-wide analysis as a critical sensitizer for TRAIL-induced apoptosis in glioblastoma cells. Nucleic Acids Res 45: 5930-5944, 2017.

14. Li LY, Xiao J, Liu Q and Xia K: Parecoxib inhibits glioblastoma cell proliferation, migration and invasion by upregulating miRNA-29c. Biol Open 6: 311-316, 2017.

15. Bing ZT, Yang GH, Xiong J, Guo L and Yang L: Identify signature regulatory network for glioblastoma prognosis by integrative mRNA and miRNA co-expression analysis. IET Syst Biol 10: 244-251, 2016. 
16. Hu HQ, Sun LG and Guo WJ: Decreased miRNA-146a in glioblastoma multiforme and regulation of cell proliferation and apoptosis by target Notch1. Int J Biol Markers 31: e270-e275, 2016.

17. Liu W, Li P, Xu Q and Wang C: Regulation of miR-181 on chemotherapy-resistant cervical cancer cells. Int J Clin Experimental Pathol 9: 4560-4565, 2016.

18. Zhou WY, Chen JC, Jiao TT, Hui N and Qi X: MicroRNA-181 targets Yin Yang 1 expression and inhibits cervical cancer progression. Mol Med Rep 11: 4541-4546, 2015.

19. Livak KJ and Schmittgen TD: Analysis of relative gene expression data using real-time quantitative PCR and the 2(-Delta Delta C(T)) method. Methods 25: 402-408, 2001.

20. Wang X, Xin Z, Xu Y and Ma J: Upregulated miRNA-622 inhibited cell proliferation, motility, and invasion via repressing Kirsten rat sarcoma in glioblastoma. Tumour Biol 37: 5963-5970, 2016.

21. Bo LJ, Wei B, Li ZH, Wang ZF, Gao Z and Miao Z: Bioinformatics analysis of miRNA expression profile between primary and recurrent glioblastoma. Eur Rev Med Pharmacol Sci 19: 3579-3586, 2015.

22. Kouri FM, Ritner C and Stegh AH: miRNA-182 and the regulation of the glioblastoma phenotype-toward miRNA-based precision therapeutics. Cell Cycle 14: 3794-3800, 2015.

23. LeBlanc VC and Morin P: Exploring miRNA-associated signatures with diagnostic relevance in glioblastoma multiforme and breast cancer patients. J Clin Med 4: 1612-1630, 2015.

24. Wu SL, Fu X, Huang J, Jia TT, Zong FY, Mu SR, Zhu H, Yan Y, Qiu S, Wu Q, et al: Genome-wide analysis of YB-1-RNA interactions reveals a novel role of YB-1 in miRNA processing in glioblastoma multiforme. Nucleic Acids Res 43: 8516-8528, 2015.

25. Das S, Kohr M, Dunkerly-Eyring B, Lee DI, Bedja D, Kent OA, Leung AK, Henao-Mejia J, Flavell RA and Steenbergen C: Divergent effects of miR-181 family members on myocardial function through protective cytosolic and detrimental mitochondrial microRNA targets. J Am Heart Assoc 6: pii: e004694, 2017.
26. Strotbek M, Schmid S, Sànchezgonzàlez I, Boerries M, Busch H and Olayioye MA: miR-181 elevates Akt signaling by co-targeting PHLPP2 and INPP4B phosphatases in luminal breast cancer. Int J Cancer 140: 2310-2320, 2017.

27. Liu J, Xing Y and Rong L: miR-181 regulates cisplatin-resistant non-small cell lung cancer via downregulation of autophagy through the PTEN/PI3K/AKT pathway. Oncol Rep 39: 1631-1639, 2018.

28. Su R, Lin HS, Zhang XH, Yin XL, Ning HM, Liu B, Zhai PF, Gong JN, Shen C, Song L, et al: MiR-181 family: Regulators of myeloid differentiation and acute myeloid leukemia as well as potential therapeutic targets. Oncogene 34: 3226-3239, 2015.

29. Ge B, Li J, Wei Z, Sun T, Song Y and Khan NU: Functional expression of CCL8 and its interaction with chemokine receptor CCR3. BMC Immunol 18: 54, 2017.

30. Lee JU, Cheong HS, Shim EY, Bae DJ, Chang HS, Uh ST, Kim YH, Park JS, Lee B, Shin HD and Park CS: Gene profile of fibroblasts identify relation of CCL8 with idiopathic pulmonary fibrosis. Respir Res 18: 3, 2017.

31. Halvorsen EC, Hamilton MJ, Young A, Wadsworth BJ, LePard NE, Lee HN, Firmino N, Collier JL and Bennewith KL: Maraviroc decreases CCL8-mediated migration of CCR5(+) regulatory $\mathrm{T}$ cells and reduces metastatic tumor growth in the lungs. Oncoimmunology 5: e1150398, 2016.

This work is licensed under a Creative Commons Attribution-NonCommercial-NoDerivatives 4.0 International (CC BY-NC-ND 4.0) License. 